\title{
The relationship between the number of circulating tumor cells and the prognosis in patients with esophageal squamous cell carcinoma
}

\author{
Zhipeng Ren", Xiaobin Hou", Zhiqiang Xue, Lianbin Zhang, Bo Wang, Jiaxin Wen, Xiangyang Chu \\ Department of Thoracic Surgery, First Medical Center, General Hospital of Chinese PLA, Beijing, China \\ Contributions: (I) Conception and design: Z Ren; (II) Administrative support: X Chu, X Hou; (III) Provision of study materials or patients: All authors; \\ (IV) Collection and assembly of data: Z Ren, X Hou; (V) Data analysis and interpretation: Z Ren; (VI) Manuscript writing: All authors; (VII) Final \\ approval of manuscript: All authors. \\ "These authors contributed equally to this work as co-first authors. \\ Correspondence to: Xiangyang Chu. Department of Thoracic Surgery, First Medical Center, General Hospital of Chinese PLA, No. 28 Fuxing Road, \\ Haidian District, Beijing 100853, China. Email: submission_301th@163.com.
}

Background: Esophageal cancer (EC) is one of the most common malignancies worldwide, with high morbidity and mortality rates. Circulating tumor cell (CTC) detection has become a novel approach in clinical study of EC. In this study, the relationship between CTCs/c-Kit expression of CTCs and the prognosis of EC patients was analyzed in EC.

Methods: A total of 43 EC patients with R0 resection were recruited for this study. The CanPatrol method was used to detect the CTC number in the peripheral blood of patients before and after operations, and the epithelial/interstitial type was classified. Multiple RNA in situ hybridization (RNA-ISH) was used to observethe c-Kit expression of CTCs. Post-operation follow-up occurred over 3 years. Logistic regression or the Cox proportional risk regression model was applied to analyze the relationship between CTC number, CTCs and disease characteristics, pathological stages and prognosis of patients with EC, and changes in CTCs before and after operations. c-Kit expression in different CTCs and the relationship between c-Kit expression and prognosis were also studied.

Results: The detection rate of CTCswas $81 \%$ (35/43). The detection rates of epithelial-, mixed- and stromal-type CTCs were 53\%, 63\%, and 33\%, respectively. The 3-year overall survival rate was $67 \%$. A CTC level of $>2$ indicated an increased risk of recurrence, metastasis, and death $(\mathrm{P}=0.018,0.002$, respectively). Following the operations, the total number of CTCs decreased in 29 cases. Of these, 6 cases were unchanged, and 8 cases demonstrated elevated CTCs. There was a significant difference in the positive rate of mixed-type CTCs before and after the operations. The rate of c-Kit expression in CTCs of EC patients was $46 \%$ pre-operation. No statistically significant correlations were found between c-Kit expression and postoperative recurrence/metastasis/survival of EC patients.

Conclusions: Preoperative CTC numbers, especially interstitial CTCs, were used as an auxiliary index in the prognosis of EC patients. The mRNA expression of c-Kit was detected in CTCs preoperatively in patients with EC, but no significant correlation between the c-Kit expression and the prognosis of EC patients was found.

Keywords: Circulating tumor cells (CTCs); c-Kit; esophageal squamous cell carcinoma; prognosis; pathology

Submitted Jun 23, 2021. Accepted for publication Aug 10, 2021.

doi: 10.21037/jgo-21-409

View this article at: https://dx.doi.org/10.21037/jgo-21-409 


\section{Introduction}

Esophageal cancer (EC) is one of the most common malignancies worldwide, with high morbidity and mortality rates (1). Although the management and treatment of patients with EC have improved over the preceding decades, the 5 -year overall survival rate remains inadequate. Various risk factors may be associated with poor prognosis of EC patients, including demographics (age, gender, or race/ ethnicity), smoking, alcohol consumption, gastroesophageal reflux disease, diet, obesity and body composition, etc. This draws urgent attention to the need for effective detection and prediction approaches before the treatment of EC. The disease often goes undiagnosed until it reaches an advanced stage due to its atypical early clinical manifestations (2). Therefore, it is necessary to identify novel targets of early diagnosis, prognosis prediction, and therapeutic strategies for patients with EC.

Recently, circulating tumor cells (CTC) regarded as the main cause of tumor metastasis or recurrence. Cancer cells that have shed from the primary tumor are able to invade into surrounding tissues, to intravasate into the bloodstream to become CTCs. At least one part of CTCs will be able to generate distant metastases. CTCs are the type of tumor cells shed from a primary or metastatic tumor and are detected in the peripheral blood. CTCs are genetically and phenotypically heterogeneous with the primary tumor, so the detection of CTCs in transit through the body by a simple blood draw may not only provide useful information for early tumor diagnosis and possible treatment strategy but may also help to update the understanding of tumor biology and eventually improve the life quality of patients with different cancers (3). Previous studies have confirmed a close correlation between high levels of CTCs and the progression of several tumors in cancers such as breast cancer (4), colorectal cancer (5), gastric cancer (6), lung cancer (7), and EC (8). This suggests that high levels of CTCs are related to a worse prognosis. Research into CTCs in EC is relatively scarce, and most of the studies lack long-term follow-up.

The Kit gene, a proto-oncogene, is proven to be involved in gastrointestinal stromal tumors (9), mast cell disease (10), melanoma (11), and so on. The overexpression of Kit is associated with poor outcomes in tumor patients. However, the relationship between the Kit gene and EC remains elusive. In the present study, we analyzed the relationship between CTC levels, epithelial/mesenchymal cell types, Kit expression, and prognosis in patients with EC. We present the following article in accordance with the REMARK reporting checklist (available at https://dx.doi.org/10.21037/ jgo-21-409).

\section{Methods}

\section{Tissue specimens}

A total of 43 EC patients who underwent surgical resection (R0 resection) from June 2016 to January 2017 were recruited. The inclusion criteria were as follows: the patients underwent R0 resection, had no history of other malignant tumors, and no history of anti-tumor treatments such as radiotherapy, chemotherapy, immunotherapy, biotherapy, etc. Patients with a history of blood transfusion were excluded. Tissue specimens were checked by routine pathological examination and classified based on the EC staging criteria released by UICC/AJCC (8th edition). Peripheral blood CTC tests were performed twice before surgery and 1 week after surgery.

Regular follow-ups were made every 3 months after surgery. The follow-up endpoint of recurrence or metastasis came about if the tumor recurred or metastasized or 3 years after surgery. The endpoint of survival evaluation was death or 3 years after surgery. All procedures performed in this study involving human participants were in accordance with the Declaration of Helsinki (as revised in 2013). The study was approved by Ethical Committee of the First Medical Center,General Hospital of Chinese PLA (No. 2021-147-03) and written informed consent was obtained from all patients.

\section{CTCs separation}

The CanPatrol@ assay (Yishan, Guangzhou)was applied to detect CTCs in peripheral blood in this study. Peripheral blood of clinical patients was collected as samples. Firstly, the red blood cells were lysed in a preservation solution, then passed through a nanofiltration membrane. Due to different cell sizes of white blood cells (smaller) and tumor cells (larger), tumor cells with larger diameters could not pass through the membrane and could not be collected. Subsequently, multiple RNA in situ hybridization (RNAISH) was used to identify and classify the collected CTCs. RNA-ISH results were observed by a semi-automatic fluorescence microscope; microscopic features of the CTC karyotype were represented in Figure 1. After the RNAISH and staining, different antibodies with specific-color fluorescence signals were used to classify the CTCs. The 

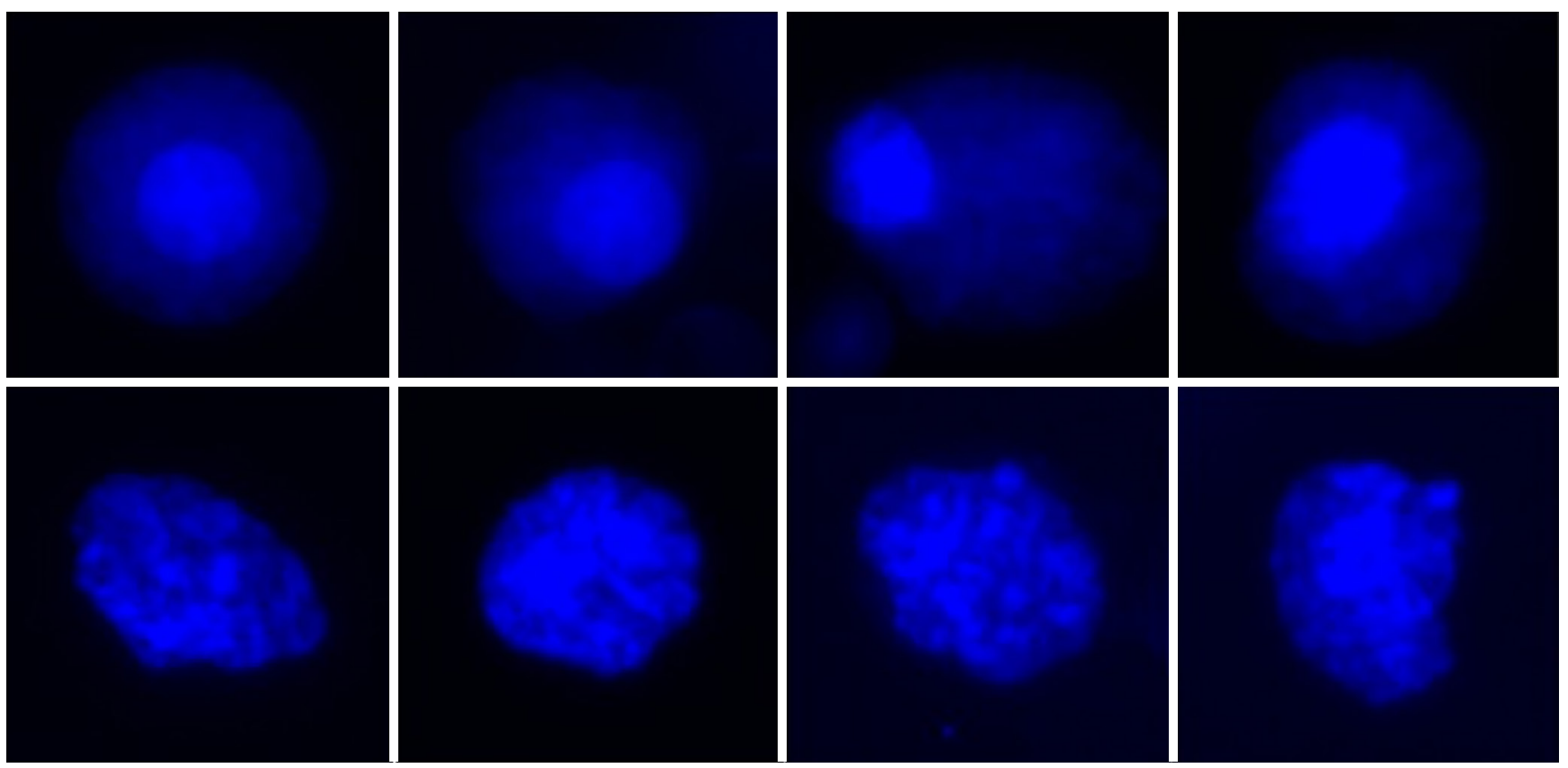

Figure 1 Microscopic features of the CTC karyotype. DAPI staining, magnification: 400×. (Row 1) A circular region with darker staining is seen in the middle of the nucleus, and the marginal region is lighter. Clear nuclear striations can be seen. (Row 2) Deep cracks with folds are seen in the nucleus with a thin karyotype. CTC, circulating tumor cell.

Table 1 Classification criteria of CTCs

\begin{tabular}{lcccc}
\hline Type & Red & Green & White & Blue \\
\hline Epithelial type & + & - & - & + \\
Interstitial type & - & + & - & + \\
Mixed type & + & + & - & + \\
\hline
\end{tabular}

CTC, circulating tumor cell.

Classification criteria of CTCs were shown in Table 1.

\section{Detection of c-Kit gene expression in CTCs}

The expression levels of c-kit in CTCs were detected after collection. Multiple probes (probe working solution) were added to in situ hybridizations of multiple RNAs. CD45 probes labeled leukocytes for reverse screening, showing the white signal under fluorescence microscope if positive; epithelial CTCs were labeled with EpCAM and CK8/18/19 probes and displayed a red signal when positive; interstitial CTCs were labeled with a Vimentin probe and Twist probe to display a green signal; the positive c-Kit probe displayed a purple signal. DAPI was used to stain the nuclei blue (Figure 2).

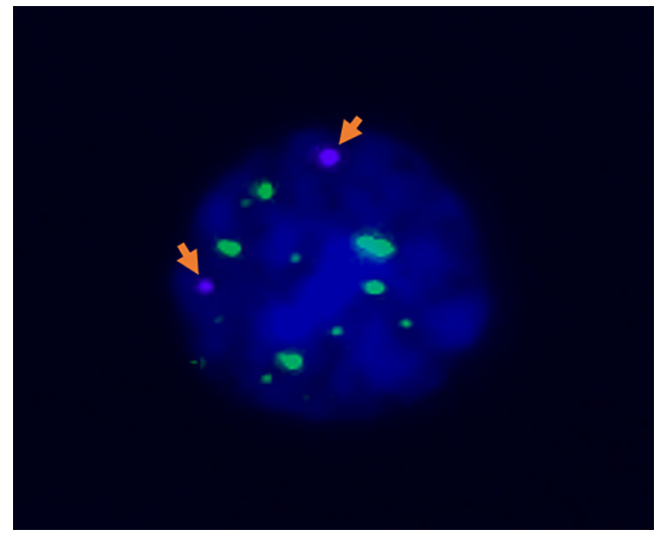

Figure 2 The expression of the c-Kit gene in the CTCs. DAPI staining, magnification: 400×. The purple fluorescent signal (arrows) indicated a positive c-Kit expression result. CTC, circulating tumor cell.

\section{Statistical analysis}

Statistical evaluation was performed using SAS software (Version 9.4, SAS Institute, Inc., Cary, North Carolina, USA). The continuous variables and the number of CTCs were expressed as mean \pm standard error or median 
(interquartile spacing), the classified variables were provided as a frequency (percentage). Associations and differences among the different parameters were analyzed using the $t$-test or Mann-Whitney $\mathrm{U}$ test, and the chi-square test or Fisher's exact test was performed for classification variables.

Multivariate logistic regression analysis was performed, and the odds ratio (OR) was adjusted for gender, family history, and other factors. The Cox proportional hazards regression model was used to calculate mortality hazard ratios and $95 \%$ confidence intervals (CIs). The KaplanMeier method and log-rank test were used for survival analyses. A P value $<0.05$ was considered as statistically significant.

\section{Results}

\section{Baseline characteristics}

A total of 43 EC patients who had undergone R0 resection were enrolled in this study, made up of 29 males (67.4\%) and 14 females $(32.6 \%)$. The mean age of patients at diagnosis was 61 years old. Among them, there were 22 (51.2\%) smokers and $21(48.8 \%)$ non-smokers. Based on postoperative pathology and TNM staging, 18 (41.9\%) patients were in stage I, $5(11.6 \%)$ were in stage II, 15 $(34.9 \%)$ were in stage III, and $5(11.6 \%)$ were in stage IV (Table 2).

\section{Detection rates of CTCs with epithelial, mixed, and stromal types in EC patients}

Among all 43 patients, 23 patients displayed epithelialtype CTCs (53\%), with the detected range from 0 to 9; 27 patients displayed mixed CTCs $(63 \%$, range from $0-7)$; interstitial CTCs were detected in 14 patients (33\%, range from 0-7) (Table 3). There were 12 patients with 0 epithelial-type CTCs, and the mixed and stromal types could not be detected.

\section{The relationship between different types of CTCs and pathological stages}

The EC patients were divided into groups based on their number of CTCs. Among these, CTCs (epithelial + mixed + interstitial) were grouped into $\leq 2$ and $>2$; CTCs of epithelial and mixed type were grouped into $\leq 1$ and $>1$, and interstitial CTCs were grouped into 0 and $>0$.

For patients with CTCs (epithelial + mixed + interstitial), logistic regression showed that the OR (95\% CI) of stage III/IV patients with more than 2 CTCs was $2.29(0.67-7.84)$ via univariate analysis, compared with the group of patients with CTCs $\leq 2$. After further adjusting for gender and family history, the detected OR (95\% CI) of stage III/IV patients with more than 2 CTCs detected was 2.55 (0.71-9.11), compared with the group of patients with CTCs $\leq 2$. Although there was no statistically significant association between the number of CTCs and disease stages, there was an upward trend in the risk of the III/IV stage in patients with more than 2 CTCs detected (Table 4).

Similarly, in patients with epithelial-type CTCs, the OR (95\% CI) of stage III/IV patients displaying more than 1 detected epithelial CTC was $0.40(0.09-1.84)$ in univariate analysis, compared with the CTCs $\leq 1$ group. After further adjustment for gender and family history, the OR (95\% CI) of stage III/IV patients with CTCs $>1$ was $0.41(0.09-1.88)$ compared with the group of CTCs $\leq 1$ (Table 4).

In univariate analysis, the OR $(95 \% \mathrm{CI})$ of stage III/IV patients with more than 1 mixed CTC was 1.87 (0.55-6.39) compared with the CTCs $\leq 1$ group. After adjusting for gender and family history, the OR (95\% CI) of stage III/IV patients with CTCs $>1$ was 1.94 (0.55-6.79) compared with the CTCs $\leq 1$ group. Although there was no statistically significant association between the number of CTCs and the disease stages, there was an upward trend in the risk of the III/IV stage in patients with more than 1 CTC detected (Table 4).

As for interstitial-type CTCs patients, the results of the univariate analysis showed the OR (95\% CI) of stage III/IV patients with CTCs $>0$ was greater than the group where CTCs $=0$, which was 1.89 (0.52-6.87). After further adjustment for gender and family history, the OR (95\% CI) of stage III/IV in patients where CTCs $>0$ were detected was $2.10(0.55-8.00)$ compared with the CTCs $=0$ group. Due to the relatively small sample size in this study, there was no significant relationship between the CTC number and disease stages. However, the risk of stage III/IV increased in patients with more than 1 interstitial CTC (Table 4).

\section{Analysis of changes in pre- and post-operation CTCs}

In the present study, we analyzed the CTCs in the peripheral blood of patients pre- and post-operation. The results were shown as follows: following operation, there were 15 patients displaying a positive result for epithelial CTCs (34.9\%); 22 patients displaying mixed CTCs (51.2\%); interstitial CTC results were positive in 11 patients $(25.6 \%)$. 
Table 2 Baseline characteristics of EC patients

\begin{tabular}{|c|c|c|c|c|}
\hline Characteristics & Total & Male & Female & $\mathrm{P}$ \\
\hline Age (year) & $61 \pm 9$ & $61 \pm 10$ & $61 \pm 7$ & 0.83 \\
\hline Smoking history & & & & $<0.001$ \\
\hline No & $21(49 \%)$ & $8(28 \%)$ & 13 (93\%) & \\
\hline Drinking history & & & & $<0.001$ \\
\hline No & $26(60 \%)$ & $12(41 \%)$ & $14(100 \%)$ & \\
\hline Yes & $17(40 \%)$ & 17 (59\%) & $0(0 \%)$ & \\
\hline Family history of EC & & & & 0.15 \\
\hline Pathological stage & & & & 0.17 \\
\hline I & $18(42 \%)$ & $11(38 \%)$ & $7(50 \%)$ & \\
\hline II & $5(12 \%)$ & $5(17 \%)$ & $0(0 \%)$ & \\
\hline III & $15(35 \%)$ & 9 (31\%) & $6(43 \%)$ & \\
\hline IV & $5(12 \%)$ & $4(14 \%)$ & $1(7 \%)$ & \\
\hline $\mathrm{T}$ & & & & 0.69 \\
\hline 1 & $18(42 \%)$ & $13(45 \%)$ & $5(36 \%)$ & \\
\hline 2 & $13(30 \%)$ & 7 (24\%) & $6(43 \%)$ & \\
\hline 1 & $13(30 \%)$ & $7(24 \%)$ & $6(43 \%)$ & \\
\hline 2 & $3(7 \%)$ & $3(10 \%)$ & $0(0 \%)$ & \\
\hline 3 & $4(9 \%)$ & $3(10 \%)$ & $1(7 \%)$ & \\
\hline M & & & & NA \\
\hline 0 & $43(100 \%)$ & $29(100 \%)$ & $14(100 \%)$ & \\
\hline G & & & & 0.21 \\
\hline 1 & $2 / 41$ (5\%) & $0 / 27(0 \%)$ & $2 / 14(14 \%)$ & \\
\hline 2 & $37 / 41(90 \%)$ & 25/27 (93\%) & $12 / 14(86 \%)$ & \\
\hline 3 & $2 / 41(5 \%)$ & $2 / 27(7 \%)$ & $0 / 14(0 \%)$ & \\
\hline
\end{tabular}

EC, esophageal cancer. 
Table 3 Detection rate of preoperative CTCs with epithelial, mixed and interstitial type

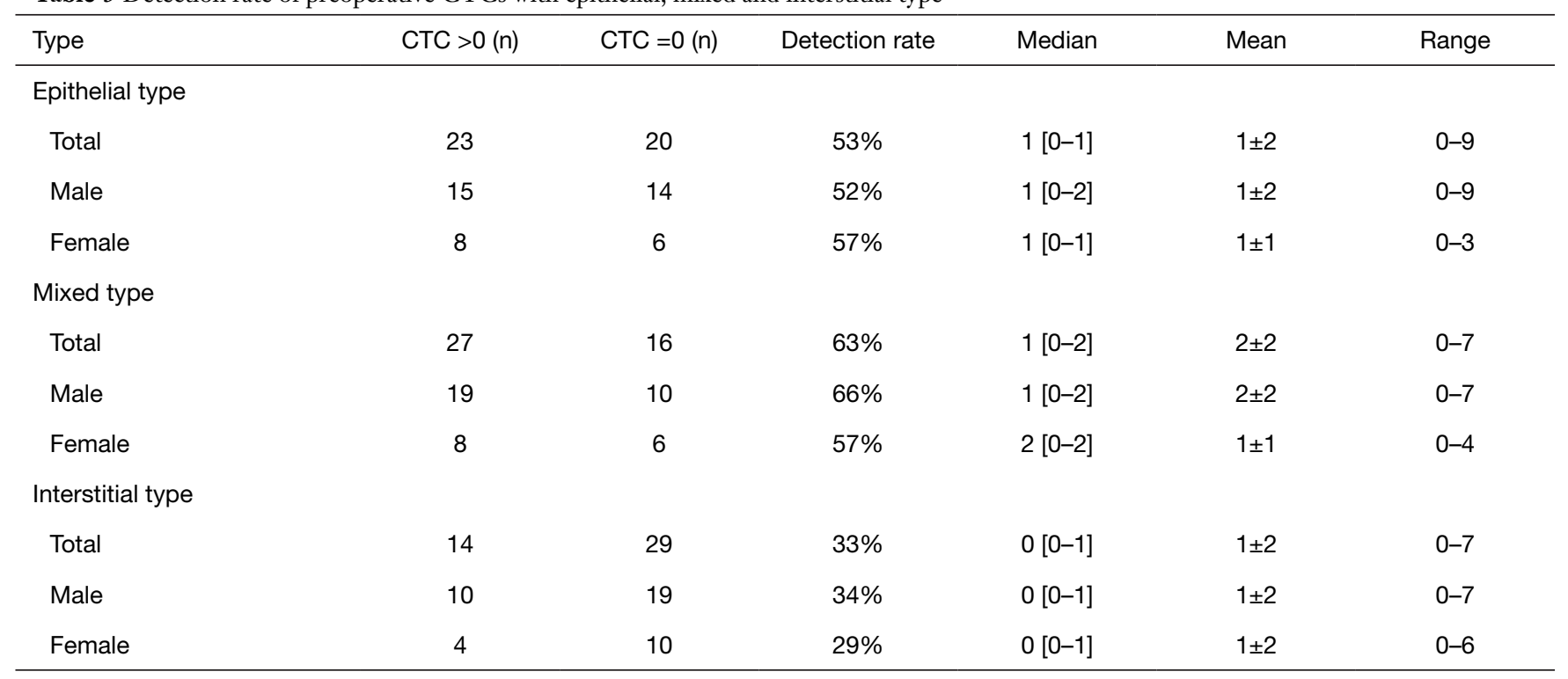

CTC, circulating tumor cell.

Table 4 The relationship between different types of CTCs and pathological stages

\begin{tabular}{|c|c|c|c|c|c|}
\hline Type & $\mathrm{n}$ & \multicolumn{2}{|c|}{ Model 1} & \multicolumn{2}{|c|}{ Model 2} \\
\hline \multicolumn{6}{|l|}{ Type 1} \\
\hline $\mathrm{CTC} \leq 2$ & 24 & 1 & - & 1 & - \\
\hline CTC >2 & 19 & $2.29(0.67-7.84)$ & 0.19 & $2.55(0.71-9.11)$ & 0.15 \\
\hline CTC $\leq 1$ & 33 & 1 & - & 1 & - \\
\hline CTC $>1$ & 10 & $0.40(0.09-1.84)$ & 0.24 & $0.41(0.09-1.88)$ & 0.25 \\
\hline \multicolumn{6}{|l|}{ Type 3} \\
\hline $\mathrm{CTC} \leq 1$ & 25 & 1 & - & 1 & - \\
\hline $\mathrm{CTC}=0$ & 29 & 1 & - & 1 & - \\
\hline CTC $>0$ & 14 & $1.89(0.52-6.87)$ & 0.33 & $2.10(0.55-8.00)$ & 0.28 \\
\hline
\end{tabular}

Type 1, epithelial + mixed + interstitial type; type 2, epithelial type; type 3, mixed type; type 4, interstitial type. Model 1: univariate analysis; Model 2: adjust gender and family history of esophageal cancer. CTC, circulating tumor cell; OR, odds ratio; $95 \% \mathrm{Cl}, 95 \%$ confidence interval.

The total number of CTC-positive patients was $30(69.8 \%)$. Among them, the positive rate of mixed-type CTCs represented a significant difference before and after surgery. Other subtypes of CTCs showed a decrease without statistical significance (Table 5).

\section{The relationship between CTC types and recurrence/ metastasis in patients}

The classification of EC patients with different types of CTCs was the same as previously described, namely, CTCs (epithelial 
Table 5 Changes in pre- and post-operation CTCs

\begin{tabular}{|c|c|c|c|c|c|c|c|}
\hline Type & \multicolumn{3}{|c|}{ Pre-operation } & \multicolumn{3}{|c|}{ Post-operation } & $\mathrm{P}$ \\
\hline Epithelial & 23 & 20 & $53.5 \%$ & 15 & 28 & $34.9 \%$ & 0.20 \\
\hline Mixed & 27 & 16 & $62.8 \%$ & 22 & 21 & $51.2 \%$ & 0.047 \\
\hline Interstitial & 14 & 29 & $32.6 \%$ & 11 & 32 & $25.6 \%$ & 0.07 \\
\hline
\end{tabular}

CTC, circulating tumor cell.

+ mixed + interstitial) were grouped into $\leq 2$ and $>2$; CTCs of epithelial and mixed type were grouped into $\leq 1$ and $>1$, and the interstitial CTCs were grouped into 0 and $>0$.

The results of Kaplan-Meier survival analysis and the logrank test showed that the risk of post-operative recurrence and metastasis was significantly increased in patients within the high CTCs group ( $\mathrm{P}=0.018$, Figure $3 A)$. As for patients with epithelial-type and mixed-type CTCs, no statistically significant difference was found in the risk of postoperative recurrence and metastasis between the two groups $(\mathrm{P}=0.53$, 0.46 , respectively; Figure $3 B, 3 C$ ). In patients with interstitialtype CTCs, the risk of postoperative recurrence and metastasis was increased in the CTCs $>0$ group $(\mathrm{P}=0.033$, Figure 3D).

\section{The relationship between CTC types and postoperative survival of patients}

Among the total 43 patients, 14 patients died 3 years later, demonstrating a 3 -year overall survival rate of $67 \%$. The group classification remained the same as in the previous method. Patients with CTCs (epithelial + mixed + interstitial) showed an increased risk of postoperative death within the high CTCs group $(\mathrm{P}=0.002$, Figure $3 E)$, with a median survival of 30 months. As for patients with epithelial-type and mixed-type CTCs, no statistically significant difference was found for the risk of post operative death between the two groups $(\mathrm{P}=0.86,0.18$, respectively; Figure $3 F, 3 G$ ). In patients with interstitial-type CTCs, there was an increased risk of postoperative death $(\mathrm{P}=0.012$, Figure $3 H)$, with a median survival of 28 months.

\section{The characteristics of c-Kit expression of CTCs in EC patients}

In all 43 patients, preoperative CTCs were tested for
c-Kit gene expression. The analyses showed there were no differences between age, gender, smoking history, drinking history, and family history in both of two groups of patients displaying c-Kit gene expression $(\mathrm{P}=0.32,0.75,0.45,0.23$ and 0.83 , respectively). The rate of positive tests for c-Kit was $46 \%(20 / 43)$. The c-Kit expression was positive in 7 cases in stage I, in 2 cases in stage II, in 9 cases in stage III and in 2 cases in stage IV. There was no difference indicated between these two groups (Table 6).

\section{The relationship between $c$-Kit expression of CTCs and tumor recurrence/survival of EC patients}

Kaplan-Meier survival analysis was performed in groups displaying both positive and negative c-Kit expression results and a log-rank test was applied for comparison between the two groups. The results showed that there was no significant difference in the risk of postoperative recurrence of tumors and metastasis between the two groups $(\mathrm{P}=0.56$, Figure 4).

\section{Discussion}

EC is a common malignant tumor that has serious implications for the survival and life quality of patients. Although a $10 \%$ increase in 5 -year survival rate was seen from 2005-2015, the overall survival rate of EC patients remains low (12). Therefore, early diagnosis, accurate treatment and comprehensive research has important clinical significance. In this study, preoperative and postoperative peripheral blood of EC patients with R0 resection was obtained to detect the number of CTCs and the epithelial/interstitial type of CTC. A three-year followup monitored EC patients and collected data to analyze the relationship between the number and type of CTCs and the clinical characteristics, pathological stage, and prognosis of 
A

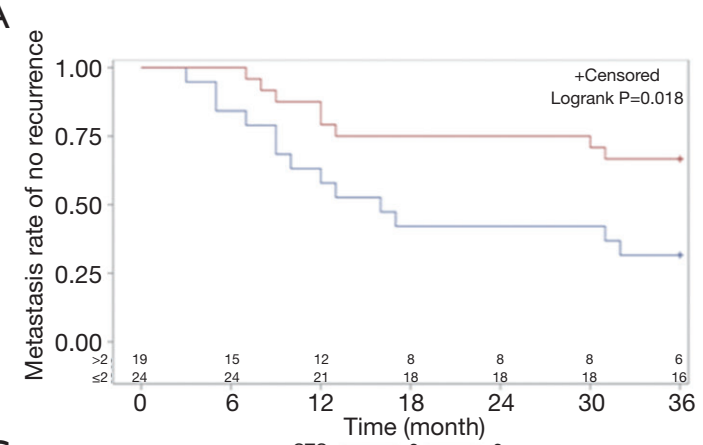

C

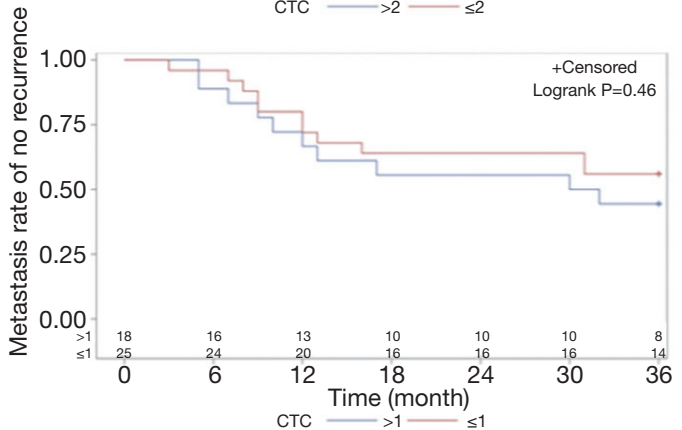

E

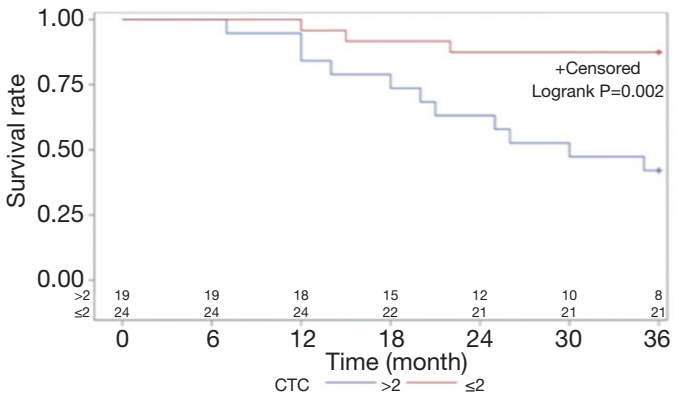

G

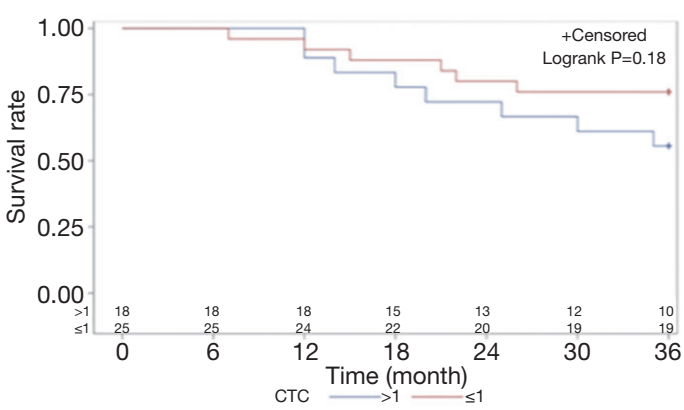

B

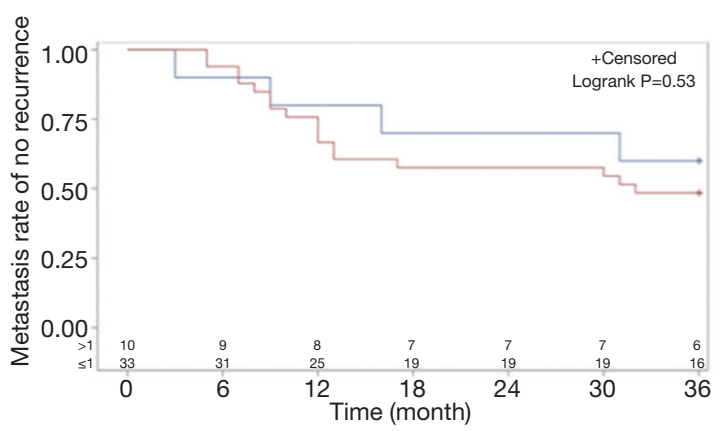

D。

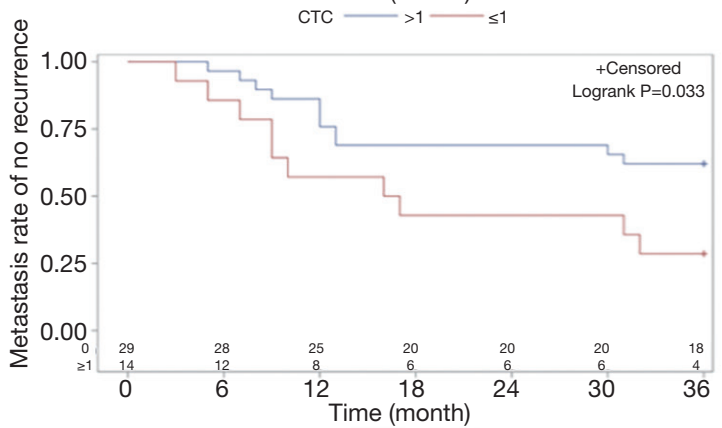

F

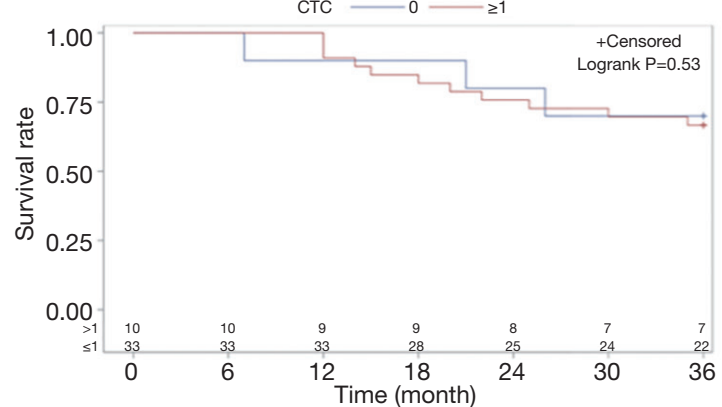

$\mathrm{H}$

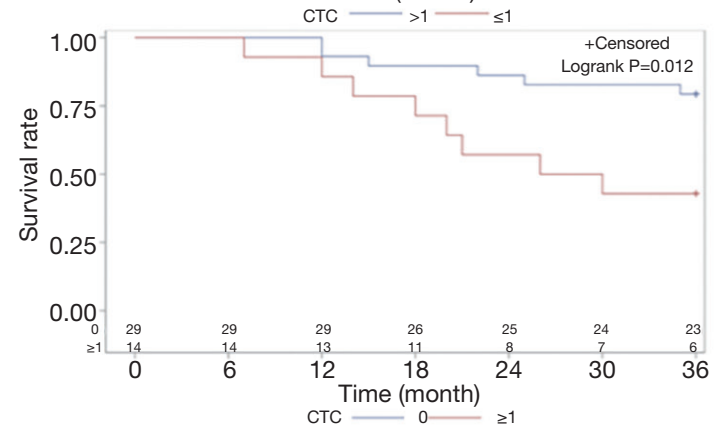

Figure 3 The relationship between CTC types and recurrence/metastasis/survival of patients. The risk of postoperative recurrence and metastasis in EC patients with CTCs (epithelial + mixed + interstitial) (A), epithelial-type (B), mixed-type (C), and interstitial-type CTCs (D); the risk of postoperative death in EC patients with CTCs (epithelial + mixed + interstitial) (E), epithelial-type (F), mixed-type (G), and interstitial-type CTCs (H). CTC, circulating tumor cell; EC, esophageal cancer.

EC patients.

We classified CTCs into the 3 subtypes of epithelial, mixed and interstitial based on their pathological features.
We found an increased risk of post-operative recurrence of tumors, metastasis, and death in the high CTC group. The detection rates of epithelial-, mixed- and interstitial- 
Table 6 Characteristics of c-Kit expression of CTCs in EC patients

\begin{tabular}{|c|c|c|c|}
\hline Characteristics & Negative & Positive & $P$ \\
\hline Age (year) & 62 & 60 & 0.32 \\
\hline Gender & & & 0.75 \\
\hline Male & $16(70 \%)$ & $13(65 \%)$ & \\
\hline Smoking history & & & 0.45 \\
\hline No & $10(43 \%)$ & $11(55 \%)$ & \\
\hline Yes & $13(57 \%)$ & $9(45 \%)$ & \\
\hline Drinking history & & & 0.23 \\
\hline Family history of EC & & & 0.83 \\
\hline No & 19 (83\%) & $16(80 \%)$ & \\
\hline Yes & $4(17 \%)$ & $4(20 \%)$ & \\
\hline Pathological stage & & & 0.64 \\
\hline 1 & $11(48 \%)$ & $7(35 \%)$ & \\
\hline II & $3(13 \%)$ & $2(10 \%)$ & \\
\hline III & $6(26 \%)$ & $9(45 \%)$ & \\
\hline IV & $3(13 \%)$ & $2(10 \%)$ & \\
\hline
\end{tabular}

CTC, circulating tumor cell; EC, esophageal cancer.
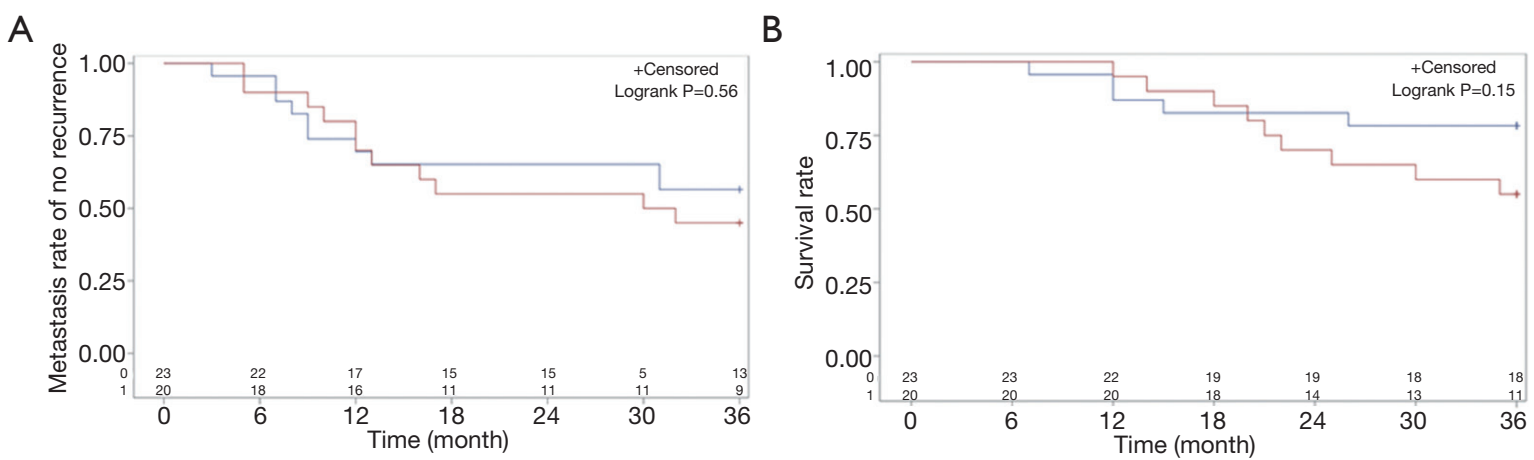

Figure 4 The relationship between c-Kit expression of CTCs and recurrence (A)/survival (B) of EC patients. Blue indicates a positive c-Kit expression result; Red indicates a negative c-Kit expression result. CTC, circulating tumor cell.

type CTCs was 53\%, 63\%, and 33\%, respectively. These results brought to light two issues in traditional epithelialCTC detection surrounding the merging of the epithelial type and mixed type together and missing the interstitial- type CTCs. Further analysis confirmed that the risk of postoperative recurrence/metastasis and death were increased in patients with CTCs $>0$ compared with the group of interstitial CTCs $=0$. 
$\mathrm{CanPatrol}^{\circledR}$ technology was applied in CTC detection of several malignancies including $\mathrm{EC}$, breast cancer, prostate cancer, colorectal cancer, gastric cancer, to name a few. It has been widely accepted that the CanPatrol ${ }^{\circledR}$ method does not only classify the subtypes and improve the detection rate of CTCs, but is also associated with the prognosis of patients with interstitial-type CTCs (13-17). Chen et al. utilised the CanPatrol ${ }^{\circledast}$ method to study the relationship between CTCs and clinical stages of EC, finding that interstitial CTCs were related to the pathological stage of EC, consistent with our study. Additionally, the number of interstitial CTCs were associated with neoadjuvant chemotherapy of EC, but no prognostic analysis was conducted (18).

Our study highlighted the necessity of CTC detection in EC. A meta-analysis conducted by $\mathrm{Li}$ et al. demonstrated that CTCs could be considered as effective indicators in evaluating the efficacy of chemotherapy and monitoring tumor recurrence in EC patients (19). Hou et al. reported that disease-free survival (DFS) and overall survival (OS) both decreased in patients where CTCs were detected, compared with EC patients where no CTCs were detected (20). Interestingly, the rate of patients testing positive for CTCs was high in patients with stage I EC after operation (16/18). We hypothesized possible explanations as follows: (I) all EC patients in stage I belonged to stage IB; (II) in CTC positive cases, there were only 3 cases where CTCs $>2$ and 1 case where interstitial CTCs $>0$; (III) in regards to prognosis, there were $2 \mathrm{EC}$ patients at stage I with recurrent tumors/ metastasis and death within 3 years, where 2 cases displayed more than 2 CTCs and 1 case of interstitial type had CTCs $>0$. CTC test may be used as an early screening method for EC in the future. Further clinical trials with larger sample sizes should be conducted to confirm the usefulness of measuring CTCs in the early diagnosis of EC.

Few studies have reported on CTC changes before and after operation. Guo et al. (21) detected CTCs of EC patients before surgery using the $\mathrm{CanPatrol}^{\circledR}$ method, showing postoperative CTC levels were generally higher than levels during the operation, and the intraoperative CTCs were higher than they were pre-operation.

Furthermore, the current study indicated that postoperative complications may lead to an increase in CTCs. However, we found that while the postoperative number of CTCs in a few patients was elevated, overall the number of CTCs in patients were reduced post-operation. The reasons for this phenomenon may have been a result of the different surgical approaches undertaken. It has been reported minimally invasive esophagectomy helped to reduce the survival rate of tumor cells in peripheral blood at the early period of postoperation, and dynamic monitoring CTC level could be used to evaluate the prognosis of EC patients.

Few studies have reported the c-Kit expression in CTCs. In this study, the c-Kit expression of preoperative CTCs was detected in patients with EC by RNA-ISH. c-Kit expression was detected in CTCs of patients with malignant melanoma and prostate cancers $(22,23)$. No other study has reported c-Kit expression in EC patients. In this study, the positive rate of c-Kit expression in CTCs was $46 \%$, but no significant correlation between c-KIT expression and prognosis of EC patients was found. This result is in line with the study published by Shang and Boone et al. $(24,25)$.

Limitations within this study were identified. Limited time and funds led to a small sample size which may have introduced bias into our results. The frequency of CTC detection was relatively low and reexamination of CTCs was not repeated during follow-ups. Therefore, we were not able to conclude that a change in CTC number could predict tumor recurrence/metastasis and long-term survival of patients. Another limitation of the study involved the analysis of only the c-Kit genes, whereas screening multiple genes at the same time could increase the probability of positive results.

In summary, the specific role of CTCs from peripheral blood in the clinical diagnosis, treatment, and disease progression of patients with EC requires further investigation. In future studies, a greater sample size and longer follow-up periods will be used to explore additional target genes involved in EC occurrence and progression, and to perform clinical trials for immunotherapy in EC. Additionally, single-cell sequencing technology could be useful in improving prospects for CTC detection.

\section{Acknowledgments}

Funding: None.

\section{Footnote}

Reporting Checklist: The authors have completed the REMARK reporting checklist. Available at https://dx.doi. org/10.21037/jgo-21-409

Data Sharing Statement: Available at https://dx.doi. org/10.21037/jgo-21-409 
Conflicts of Interest: All authors have completed the ICMJE uniform disclosure form (available at https://dx.doi. org/10.21037/jgo-21-409). The authors have no conflicts of interest to declare.

Ethical Statement: The authors are accountable for all aspects of the work in ensuring that questions related to the accuracy or integrity of any part of the work are appropriately investigated and resolved. The study was approved by Ethical Committee of PLA General Hospital (No. 2021-147-03) and written informed consent was obtained from all patients. All procedures performed in this study involving human participants were in accordance with the Declaration of Helsinki (as revised in 2013).

Open Access Statement: This is an Open Access article distributed in accordance with the Creative Commons Attribution-NonCommercial-NoDerivs 4.0 International License (CC BY-NC-ND 4.0), which permits the noncommercial replication and distribution of the article with the strict proviso that no changes or edits are made and the original work is properly cited (including links to both the formal publication through the relevant DOI and the license). See: https://creativecommons.org/licenses/by-nc-nd/4.0/.

\section{References}

1. Enzinger PC, Mayer RJ. Esophageal cancer. N Engl J Med 2003;349:2241-52.

2. Short MW, Burgers KG, Fry VT. Esophageal Cancer. Am Fam Physician 2017;95:22-8.

3. Kulemann B, Liss AS, Warshaw AL, et al. KRAS mutations in pancreatic circulating tumor cells: a pilot study. Tumour Biol 2016;37:7547-54.

4. Bidard FC, Proudhon C, Pierga JY. Circulating tumor cells in breast cancer. Mol Oncol 2016;10:418-30.

5. Nanduri LK, Hissa B, Weitz J, et al. The prognostic role of circulating tumor cells in colorectal cancer. Expert Rev Anticancer Ther 2019;19:1077-88.

6. Thanh Huong P, Gurshaney S, Thanh Binh N, et al. Emerging Role of Circulating Tumor Cells in Gastric Cancer. Cancers (Basel) 2020;12:695.

7. Maly V, Maly O, Kolostova K, et al. Circulating Tumor Cells in Diagnosis and Treatment of Lung Cancer. In Vivo 2019;33:1027-37.

8. Hoeppner J, Kulemann B. Circulating Tumor Cells in Esophageal Cancer. Oncol Res Treat 2017;40:417-22.

9. Kang W, Zhu C, Yu J, et al. KIT gene mutations in gastrointestinal stromal tumor. Front Biosci (Landmark Ed) 2015;20:919-26.

10. Wilcock A, Bahri R, Bulfone-Paus S, et al. Mast cell disorders: From infancy to maturity. Allergy 2019;74:53-63.

11. Woodman SE, Davies MA. Targeting KIT in melanoma: a paradigm of molecular medicine and targeted therapeutics. Biochem Pharmacol 2010;80:568-74.

12. Zeng $\mathrm{H}$, Chen $\mathrm{W}$, Zheng R, et al. Changing cancer survival in China during 2003-15: a pooled analysis of 17 population-based cancer registries. Lancet Glob Health 2018;6:e555-67.

13. Zhang S, Wu T, Peng X, et al. Mesenchymal phenotype of circulating tumor cells is associated with distant metastasis in breast cancer patients. Cancer Manag Res 2017;9:691-700.

14. Yang YJ, Kong YY, Li GX, et al. Phenotypes of circulating tumour cells predict time to castration resistance in metastatic castration-sensitive prostate cancer. BJU Int 2019;124:258-67.

15. Wu F, Zhu J, Mao Y, et al. Associations between the Epithelial-Mesenchymal Transition Phenotypes of Circulating Tumor Cells and the Clinicopathological Features of Patients with Colorectal Cancer. Dis Markers 2017;2017:9474532.

16. Liu DG, Xue L, Li J, et al. Epithelial-mesenchymal transition and GALC expression of circulating tumor cells indicate metastasis and poor prognosis in non-small cell lung cancer. Cancer Biomark 2018;22:417-26.

17. Cheng B, Tong G, Wu X, et al. Enumeration And Characterization Of Circulating Tumor Cells And Its Application In Advanced Gastric Cancer. Onco Targets Ther 2019;12:7887-96.

18. Chen W, Li Y, Yuan D, et al. Practical value of identifying circulating tumor cells to evaluate esophageal squamous cell carcinoma staging and treatment efficacy. Thorac Cancer 2018;9:956-66.

19. Li Y, Wu G, Yang W, et al. Prognostic value of circulating tumor cells detected with the CellSearch system in esophageal cancer patients: a systematic review and metaanalysis. BMC Cancer 2020;20:581.

20. Hou J, Zou K, Yang C, et al. Clinicopathological and prognostic significance of circulating tumor cells in patients with esophageal cancer: a meta-analysis. Onco Targets Ther 2018;11:8053-61.

21. Guo X, Wu YZ, Jia LF, et al. Effects of minimally invasive versus open esophagectomy on circulating tumor cells in patients with esophageal cancer. Nan Fang Yi Ke Da Xue Xue Bao 2018;38:318-23. 
22. Sakaizawa K, Goto Y, Kiniwa Y, et al. Mutation analysis of BRAF and KIT in circulating melanoma cells at the single cell level. Br J Cancer 2012;106:939-46.

23. Russo GI, Bier S, Hennenlotter J, et al. Expression of tumour progression-associated genes in circulating tumour cells of patients at different stages of prostate cancer. BJU Int 2018;122:152-9.

24. Shang L, Liu HJ, Hao JJ, et al. A panel of overexpressed

Cite this article as: Ren Z, Hou X, Xue Z, Zhang L, Wang B, Wen J, Chu X. The relationship between the number of circulating tumor cells and the prognosis in patients with esophageal squamous cell carcinoma. J Gastrointest Oncol 2021;12(4):1265-1276. doi: 10.21037/jgo-21-409 proteins for prognosis in esophageal squamous cell carcinoma. PLoS One 2014;9:e111045.

25. Boone J, van Hillegersberg R, Offerhaus GJ, et al. Targets for molecular therapy in esophageal squamous cell carcinoma: an immunohistochemical analysis. Dis Esophagus 2009;22:496-504.

(English Language Editors: B. Maizey and J. Chapnick) 